
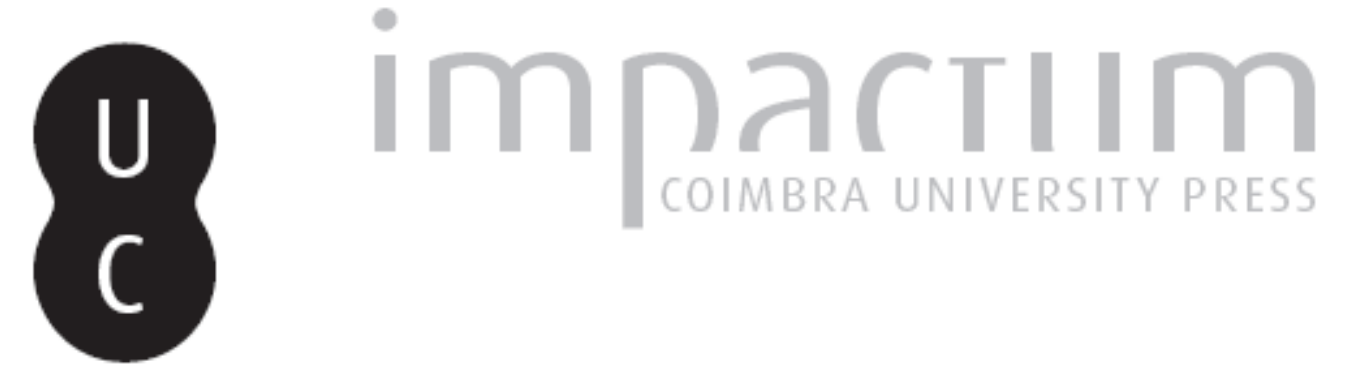

\title{
Appunti per un'analisi comparata del fenomeno della canzone impegnata degli anni 60 e 70 in Portogallo e Italia
}

\author{
Autor(es): $\quad$ Merlini, Dea
}

Publicado por: Imprensa da Universidade de Coimbra

URL persistente:

URI:http://hdl.handle.net/10316.2/42411

DOI:

DOI:https://doi.org/10.14195/0870-8584_9_5

Accessed : $\quad$ 26-Apr-2023 12:00:06

A navegação consulta e descarregamento dos títulos inseridos nas Bibliotecas Digitais UC Digitalis, UC Pombalina e UC Impactum, pressupõem a aceitação plena e sem reservas dos Termos e Condições de Uso destas Bibliotecas Digitais, disponíveis em https://digitalis.uc.pt/pt-pt/termos.

Conforme exposto nos referidos Termos e Condições de Uso, o descarregamento de títulos de acesso restrito requer uma licença válida de autorização devendo o utilizador aceder ao(s) documento(s) a partir de um endereço de IP da instituição detentora da supramencionada licença.

Ao utilizador é apenas permitido o descarregamento para uso pessoal, pelo que o emprego do(s) título(s) descarregado(s) para outro fim, designadamente comercial, carece de autorização do respetivo autor ou editor da obra.

Na medida em que todas as obras da UC Digitalis se encontram protegidas pelo Código do Direito de Autor e Direitos Conexos e demais legislação aplicável, toda a cópia, parcial ou total, deste documento, nos casos em que é legalmente admitida, deverá conter ou fazer-se acompanhar por este aviso.

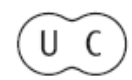




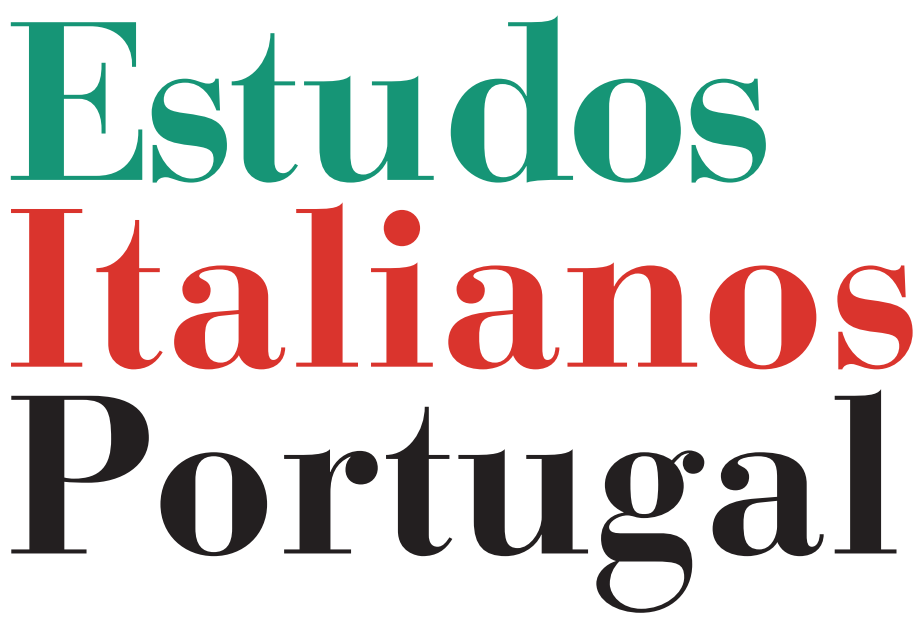

Instituto

Italiano

de Cultura

de Lisboa

Nova Série

$\mathbf{N}^{\circ} 9$ 


\title{
APPUNTI PER UN'ANALISI COMPARATA DEL FENOMENO DELLA CANZONE IMPEGNATA DEGLI ANNI 60 E 70 IN PORTOGALLO E ITALIA
}

\author{
Dea Merlini*
}

DISCIPLINE COME LA CRITICA STORIOGRAFICA e la sociologia hanno da sempre guardato con attenzione al valore documentario delle espressioni artistiche (prima fra tutte la letteratura ma anche, più recentemente, il cinema), ma tra queste poco spazio è stato dato alla musica, soprattutto a quel tipo di musica di cui qui ci occuperemo: la canzone, intesa come struttura costituita dall'unione tra una melodia e un testo.

Non solo la canzone è stata a lungo ignorata come fonte per la ricerca storica, ma in molti casi è stata esclusa anche dall'ambito degli studi artistico-letterari, considerata un mero fenomeno commerciale ${ }^{1}$ e guardata con quella sufficienza che ben esprimono etichette come 'musica leggera', con cui in Italia si usa identificare, paradossalmente, anche il ramo della canzone cosiddetta 'impegnata'. Recentemente, però, assistiamo al formarsi di un nuovo atteggiamento criti-

* Nata a Cecina il 22 luglio 1990. Laureata presso l'Università di Pisa con una tesi sul recupero della tradizione della poesia popolare nelle Quadras di Fernando Pessoa. Frequenta il corso di laurea magistrale in Letterature e Filologie Euroamericane, presso l'Università di Pisa, ed è allieva della SNS. Ha collaborato con le riviste Il Ponte e Rifrazioni, pubblicando analisi critiche di film. Nell'anno 2013 ha svolto tre mesi di Erasmus placement presso l'UC.

${ }^{1}$ Sono celebri le critiche mosse da Adorno alla pop music. 
co nei confronti della pop music, che viene proprio dagli ambienti della sociologia e della storia culturale, oggi molto più inclini a riconoscerne l'eccezionale valore documentario ${ }^{2}$. Il presente articolo vuole inserirsi in una prospettiva di ricerca che fa dei fatti culturali un elemento non meramente sovrastrutturale, ma strutturante rispetto ai processi economico-politici di una società. Alla luce di ciò, accogliamo in pieno la sollecitazione di Marco Peroni a considerare la musica leggera come 'fonte di storia', in virtù del suo essere prima di tutto 'agente di storia'.

Date tali premesse, cercheremo di condurre una prima e parziale analisi delle caratteristiche della musica 'impegnata' degli anni 70 in Italia e in Portogallo, inquadrandola all'interno dei momenti storici in cui si inserisce nei due Paesi, sempre nella prospettiva di trovare nuovi angoli da cui gettare uno sguardo sui fatti storici stessi: nello specifico, i movimenti sociali e politici che hanno costituito il sostrato della Rivoluzione dei garofani in Portogallo e l'ondata di contestazioni che va dal movimento del 68 a quello del 77 in Italia. Si tratta di due contesti storici molto differenti e solo in minima parte paragonabili, ma quello che conta ai fini della nostra ricerca è che sono entrambi momenti densi di rivolgimenti sociali e politici, cui il mondo della musica ha risposto con un tentativo di trasformare la canzone da strumento di evasione a mezzo di veicolazione di contenuti più 'seri' e politicamente significativi.

Veniamo ora a tracciare in maniera molto succinta questi due contesti.

In Italia la 'canzone impegnata' vive il suo periodo aureo tra gli anni 60 e 70. Ma che cosa significa 'canzone impegnata'? O meglio, come questa formula si declina all'interno

${ }^{2}$ Si vedano in proposito M. Peroni, Il nostro concerto. La storia contemporanea tra musica leggera e canzone popolare (Le scienze del tempo), Milano, Bruno Mondadori, 2005; M. Santoro, Effetto Tenco. Genealogia della canzone d'autore, Bologna, Il Mulino, 2010; S. Pivato, Bella ciao. Canto e politica nella storia d'Italia, Roma, Laterza, 2005. 
dell'universo musicale degli anni tra il boom economico e l'esplosione delle contestazioni del 68 e del 77 ?

Col termine 'impegnata' in Italia si possono definire almeno due generi di canzone ben distinti tra loro: la canzone propriamente 'militante' e la 'canzone d'autore'. Entrambi questi mondi percepiscono se stessi e sono percepiti come 'impegnati'; laddove l'impegno può andare dalla rivendicazione della libertà della ricerca estetica a un sostegno effettivo ai movimenti politici di quella fase storica.

Iniziamo dalla canzone d'autore. Si tratta di una categoria molto labile, all'interno della quale sono stati inseriti gli artisti più disparati, tanto che risulta pressoché impossibile stilare una lista di coloro che possono a rigore definirsi 'cantautori'. Il termine, usato per la prima volta da Ennio Melis e Vincenzo Micocci (della RCA) nel 1960, per identificare commercialmente quei cantanti sorti sulla scorta di Domenico Modugno, contemporaneamente autori e interpreti delle proprie canzoni (Meccia, Fidenco, Vianello), troverà la sua massima fortuna solo più tardi, con il Club Tenco, nato sulla scia del suicidio di Luigi Tenco.

Fondato nel 1972, a Sanremo, su iniziativa di Amilcare Rampaldi, il Club Tenco si costituisce fin da subito come scena musicale indipendente, alternativa al mondo della canzone che si convogliava intorno al Festival di Sanremo; quello stesso mondo contro cui Tenco aveva dichiarato di voler protestare proprio attraverso il suicidio ${ }^{3}$. Le Rassegne organizzate dal Club al Teatro Ariston (non ancora sede ufficiale del Festival) diventeranno uno spazio insostituibile per molti cantanti emergenti; uno spazio in cui mettere a frutto il proprio talento poetico-musicale e costruire una rete di relazioni che li porterà a sviluppare la consapevolezza di far parte di

\footnotetext{
${ }^{3}$ Luigi Tenco aveva partecipato all'edizione del Festival del 1967 con la canzone Ciao amore, ciao, venendo eliminato già nella prima serata. La notte tra il 26 e il 27 gennaio, fu trovato morto all'Hotel Savoy, accanto a una rivoltella e a un biglietto in cui si spiegavano le ragioni dell'atto.
} 
una categoria di artisti con caratteristiche condivise e assolutamente nuove rispetto al panorama della canzone italiana a loro precedente e coevo. ${ }^{4}$

Marco Santoro ha sottolineato l'importanza del suicidio di Tenco per la definizione della categoria di 'cantautore', avvenuta dopo 1967; definizione che finirà per scavalcare abbondantemente il significato primario del termine, fino ad assumere un'aurea di artisticità e intellettualità quasi sacrali. Con l'atto estremo e 'romantico' del suicidio e con l'indicazione esplicita, nel biglietto di addio, di interpretarlo come una protesta contro il futile e opportunistico mondo della canzone leggera ufficiale, Tenco apriva la strada a un nuovo modo di concepire l'attività artistica. Nasceva una tipologia di canzone che non si proponeva più di assecondare i gusti vigenti a fini di successo commerciale, ma di veicolare contenuti nuovi e 'sinceri', espressione autentica di temperamenti sensibili e intelligenti, naturalmente ribelli alle verità del senso comune e ai facili valori sponsorizzati dai poteri forti. In tale contesto la coincidenza tra autore e interprete (espressa dall'accezione originaria del termine) era condizione necessaria ma non sufficiente per l'ottenimento della qualifica di 'cantautore'. Erano indispensabili, a tal proposito, un coinvolgimento personale forte rispetto ai temi trattati e una notevole complessità artisticocognitiva dei testi delle canzoni.

Se di valore artistico e impegno intellettuale la canzone d'autore rappresentava fin dalla sua comparsa un esempio indiscutibile, diverso era il caso dell'intervento più propriamente politico, che in quegli anni non poteva che esplicitarsi in una presa di posizione a favore o contro i movimenti che stavano scuotendo l'Italia. Se infatti la maggior parte dei più celebrati cantautori partecipò del clima di politicità diffusa caratteristico degli anni 60 e 70, i loro rapporti con i movimenti politici furono tutt'altro che facili. Non parliamo dei

\footnotetext{
${ }^{4}$ Queste notizie si ricavano da M. Santoro, cit.
} 
partiti, perlopiù estranei - quando non apertamente ostili - ai rivolgimenti di quegli anni, ma di quella galassia di collettivi studenteschi, movimenti operai, gruppi femministi, che tra gli anni 60 e 70 si resero protagonisti delle azioni più disparate (dalle occupazioni delle università ai blocchi di produzione delle fabbriche, dall'autoriduzione delle bollette agli espropri proletari, gli scioperi selvaggi, le performances degli 'Indiani Metropolitani', le radio libere, fino ad arrivare alla lotta armata vera e propria) e che nel 77 confluirà intorno alla cosiddetta 'Area dell'Autonomia'.

Se cantanti come De André, De Gregori, Guccini, Gaber e molti altri si mostreranno ideologicamente vicini alle pratiche del Movimento e se le loro canzoni compariranno in manifestazioni, festival indipendenti, concerti di appoggio a vari gruppi della Sinistra extraparlamentare (Lotta Continua, Potere Operaio etc.) e nelle radio libere, i loro rapporti con l'universo militante, saranno molto complicati e da questo universo riceveranno pesanti attacchi.

È emblematico l'episodio della contestazione a De Gregori avvenuta al Palalido il 2 aprile 1976. Si tratta del primo grosso concerto di un artista abituato ai circuiti indipendenti della musica, che qui si trova per la prima volta catapultato in un palazzetto dello sport riempito di più di 6000 persone, che urlano il suo nome e applaudono prima ancora che inizi a cantare. Durante il concerto, alcuni autonomi salgono sul palco e si appropriano del microfono per leggere un comunicato contro l'arresto di un militante a Padova. Lamentano la presenza di fascisti all'interno del pubblico e attaccano il cantautore per essersi reso complice di un evento pesantemente commercializzato, per di più accessibile attraverso l'acquisto di un biglietto. ${ }^{5}$ Riportiamo qui di seguito uno stralcio

\footnotetext{
${ }^{5}$ La questione non è banale se si pensa che tra le prime battaglie dell'Ala Creativa' dell'Autonomia c'è quella contro il costo dei biglietti per i concerti, in nome del libero (e gratuito) accesso alla cultura. Va detto poi che la polemica era già stata aperta pochi mesi prima, dal rifiuto di De Gregori di suonare a una festa di Lotta Continua.
} 
dell'articolo uscito il giorno seguente sul Corriere della Sera, a firma Fegiz:

$\mathrm{Al}$ microfono si alternano volti lombrosiani e giovani che sembrano colti da raptus isterico. "Suona per i lavoratori, non ti mettere in tasca i soldi". "Quanto hai preso stasera?" urla un giovane. "Credo un milione e due... - sussurra con un filo di voce De Gregori -, ma poi c’è la Siae...". "Se sei un compagno, non a parole ma a fatti, lascia qui l'incasso", ribattono. Prende la parola un uomo con la barba bianca, d'età indefinibile: "La rivoluzione non si fa con la musica. Prima si fa la rivoluzione, poi si potrà pensare alle arti o alla musica. Lo diceva anche Majakowskij che era un vero rivoluzionario e si è suicidato. Suicidati anche tu!"6

Sorvolando su toni e contenuti specifici delle recriminazioni e tenendo comunque presente il chiaro intento del giornalista di dipingere a tinte il più possibile fosche i responsabili della contestazione, emerge un'insofferenza verso un cantautore 'vendutosi al mercato', cui si chiede di fare ammenda del fatto di essersi piegato a una dinamica da 'grande evento' (con sostanziosi introiti economici), tramite una dimostrazione pubblica di fedeltà al Movimento, mostrando, cioè, di porre gli obiettivi di quest'ultimo al di sopra dei propri. Ma quello che emerge è parimenti che, di fronte all'uscita del cantautorato dai circuiti indipendenti e dilettantistici, si pone la questione del difficile rapporto di una categoria di artisti che nasce in contrapposizione alla riduzione della musica a fatto commerciale, con la necessità di agire all'interno di dinamiche commerciali per il raggiungimento di un pubblico più vasto ${ }^{7}$.

Il crescere della tensione condurrà alla rinuncia di vari cantautori a obiettivi consapevolmente politici, esemplificata

\footnotetext{
${ }^{6}$ Ricaviamo il testo da M. Santoro, cit., p. 181.

${ }^{7}$ Anche Tenco aveva partecipato a Sanremo con l'obiettivo di allargare il suo pubblico.
} 
da canzoni come L'avvelenata di Francesco Guccini (1976) e Cantautore (1976) e Sono solo canzonette (1980) di Edoardo Bennato.

I cantautori si percepiscono e sono percepiti prima di tutto come 'artisti', che possono occasionalmente parlare di politica; non come militanti che esprimono attraverso l'arte il proprio contributo a una lotta in cui sono pienamente inseriti. Tenco stesso aveva dato indicazioni chiare in tal senso:

Io faccio anzitutto il cantante. [...] Cioè, io canto non perché mi interessa protestare e quindi lo faccio cantando: io canto, ripeto, perché mi piace la musica. Da bambino prima ancora di sapere che cos'era la protesta, io avevo una chitarra in casa con la quale suonavo. ${ }^{8}$

Ma in quegli anni in Italia, accanto alla canzone d'autore, esiste anche una vera e propria canzone militante. In essa si possono includere varie esperienze riconducibili alla cosiddetta 'Ala Creativa' dell'Autonomia', che trovava espressione in riviste come Re Nudo, Linus e Muzak e nell'organizzazione di iniziative musicali tra cui rimarrà celebre il Festival di Parco Lambro (tra il 74 e il 76). Estremamente significative, per la canzone militante, sono le esperienze di Cantacronache e del Nuovo Canzoniere Italiano.

I Cantacronache sono un collettivo torinese sorto nel 1958, anch'esso in aperta polemica col mondo sanremese, cui vuole contrapporre un tipo di canzone 'impegnata'. Ma per loro il concetto di 'impegno' in musica non ha tanto a che vedere con un rapporto più autentico con i testi delle proprie canzoni, quanto con la riscoperta del patrimonio del canto sociale italiano (di matrice popolare), da cui si traeva spunto anche per creare canzoni nuove, capaci di raccontare

\footnotetext{
${ }^{8}$ M. Peroni, cit., p. 115.

${ }^{9}$ Uno tra tutti Gianfranco Manfredi, autore di uno dei veri e propri inni dell'Autonomia: Ma chi ha detto che non c’è (1976).
} 
in modo empatico e privo di patetismi la realtà del proletariato urbano e di denunciare le aporie di uno stato democratico di cui si sottolineava la continuità, sotto molti aspetti, col Fascismo. Obiettivo dell'operazione era provocare un risveglio della combattività sociale ${ }^{10}$.

Dopo la chiusura di questa esperienza (nel 1962), a raccoglierne il testimone sarà il gruppo del Nuovo Canzoniere Italiano. Sorto nel 1961 su iniziativa di Gianni Bosio e Roberto Leydi, opera in collaborazione con le edizioni Avanti e l'Istituto Ernesto de Martino (per la conoscenza critica e la presenza alternativa del mondo popolare e proletario) ${ }^{11}$. Ne nasceranno le edizioni dei Dischi del sole e spettacoli come Bella ciao (1964). Il primato delle ragioni della lotta su quelle dell'arte traspare chiaramente nella decisione collettiva di chiudere quell'esperienza stessa, una volta preso atto che, nelle parole di Bosio, "il modo di attaccare la città capitalistica non è quello di creare nuove canzoni" 12 .

Si tratta di una situazione agli antipodi rispetto a quella dei cantautori, il confronto con i quali servirà a questi interpreti a delimitare, per contrapposizione, i confini della propria attività artistica. Dice infatti Ivan della Mea, sul fallimento degli obiettivi del gruppo:

Venendo a mancare il senso di un'operazione politico-culturale complessiva eravamo diventati anche noi dei cantautori, che ottenevano poi

${ }^{10}$ Del gruppo facevano parte compositori come Sergio Liberovici, Ivan della Mea, Fausto Amodei, Michele Straniero, Emilio Jona, Margot Galante Garrone, nonché scrittori del calibro di Franco Fortini e Italo Calvino.

${ }^{11} \mathrm{La}$ collaborazione stretta col mondo della canzone popolare sarà propria anche dei cantores portoghesi e le ballate di José Afonso nasceranno proprio sulla scorta di artisti come Edmundo de Bettencourt, che avevano tentato di rivoluzionare il fado di Coimbra a partire da una sua contaminazione con la canzone folklorica.

${ }^{12}$ Da una conversazione tra Gianni Bosio, Cesare Bermani, Claudio Bernieri, Lorenza Bordes, Milano, 14 aprile 1970. La ricaviamo da N. Balestrini-P. Moroni, L'orda d'oro 1968-1977. La grande ondata rivoluzionaria e creativa, politica ed esistenziale, Milano, Feltrinelli, 2008, p. 90. 
magari al Festival nazionale dell'Unità a Firenze il massimo del successo con trenta-quarantamila ascoltatori. Mentre alcuni miei compagni dicevano: "Finalmente ce l'abbiamo fatta", io dissi "Abbiamo perso". ${ }^{13}$

Il cantautorato assurge qui a simbolo di una canzone ridotta a fatto puramente estetico, sentito come politicamente neutro, in cui anche la protesta più dura diventa una 'cosa da artisti', espressione di singoli temperamenti eccezionali ed eccentrici.

In Portogallo la situazione è molto diversa. I cosiddetti cantores de intervenção (anche detti cantores de Abril) iniziano a operare sotto una dittatura già morente, sulla scia di una stagione di malcontenti che ha visto nell'esplodere del movimento universitario, uno dei suoi momenti salienti ${ }^{14}$.

Siamo negli anni 60 e il Paese si trova di fronte a un boom economico inizialmente promosso dallo stesso regime di Salazar (con gli incentivi alle industrie, al commercio, al turismo e con l'entrata del Portagallo nell'EFTA). Nei confronti di questo sviluppo il regime si trovò ben presto come il dottor Frankenstein di fronte alla sua creatura: aveva messo in moto un processo di espansione di tipo capitalistico destinato a ritorcerglisi contro, nel momento in cui i nuovi interessi commerciali e finanziari non potevano che sentirsi stretti nelle maglie di uno stato che faceva del cristianesimo più tradizionale e dell'esaltazione del carattere rurale dei portoghesi il perno della sua retorica e che, soprattutto, intratteneva con le proprie colonie un rapporto di sfruttamento brutale e improduttivo, ben diverso dalle raffinate forme di assoggettamento che il neocolonialismo assumeva nelle più

${ }^{13}$ Da una testimonianza orale di Ivan della Mea resa a Fabrizio Borsella, Milano, 23-7-1992, ib., p. 100.

${ }^{14}$ Per le informazioni sulla canzone di protesta portoghese si rinvia a E. Raposo, Cantores de Abril. Entrevistas a cantores e outros protagonistas do 'canto de intervenção', Lisboa, Colibri, 2014, 2. a ed. rev., e Canti rivoluzionari portoghesi, a cura di L. Settimelli, L. Falavolti, Roma, Newton Compton, 1977. 
potenti democrazie occidentali. Il Paese viveva su di sé una serie di contraddizioni che porteranno allo sfociare della rivolta e all'abbattimento del regime.

In questa congiuntura, il movimento studentesco (molto diverso da quello italiano degli stessi anni, in quanto più fortemente legato a strutture prettamente universitarie) svolse un ruolo fondamentale nella perdita di consensi che porterà al collasso dello stato. In particolare le due crisi del $62 \mathrm{e}$ del 69 avevano dimostrato come il mondo universitario non fosse disposto a rinunciare ai propri spazi di autonomia. Lo svuotamento di fatto del potere delle associazioni accademiche, i tentativi di proibire la realizzazione dell'annuale Dia do estudante (nel 62) e l'attacco ai dirigenti del movimento associativo (otto di loro sospesi dall'Università di Coimbra per aver organizzato una controinaugurazione dell'edificio di Matematica, nell'aprile del 69) avevano messo in moto una serie di proteste che videro il loro picco nell'istituzione del luto académico all'Università di Coimbra (1969): il blocco degli esami e la trasformazione delle lezioni in aule di discussione politica.

All'interno di questi movimenti nasce e si sviluppa un nuovo modo di intendere la canzone, anche qui in contrapposizione alla 'canzone leggera' ufficiale. Ma esso si caratterizza immediatamente per la necessità di esprimere in forme artisticamente adeguate una protesta politica che urgeva di diventare pubblica.

Ne scaturiranno le esperienze di artisti come Adriano Correia de Oliveira, Francisco Fanhais, Francisco Naia, José Barata Moura, José Jorge Letria, José Mário Branco, José Niza, Luís Cília, Manuel Freire, Mário Vieira de Carvalho, Sérgio Godinho, Tino Flores e soprattuto José Afonso (detto Zeca).

I cantores de Abril, come i cantautori italiani, proponevano un tipo di canzone che non si appiattisse sulle dinamiche del mercato e che, dall'incontro con la poesia, traesse linfa vitale per l'incremento della propria complessità artistico-cognitiva; 
poesia che in alcuni casi era di autoria dello stesso interprete, mentre in altri era vera e propria poesia scritta, solo successivamente musicata ${ }^{15}$. Questo sodalizio tra canzone e poesia costituisce, nelle parole di Manuel Alegre, "o verdadeiro vanguardismo estético português"16.

Tornando indietro, si è detto che la canzone de intervenção nasce all'interno dei movimenti universitari e in funzione di questi. Di ciò sono testimonianza numerose dichiarazioni degli stessi autori. Ne riportiamo soltanto alcune:

José Jorge Letria:

Eu quando entro na Faculdade em 68, por um lado apanho o impulso do Maio de 68, e depois logo nesse ano, na viragem, apanho a crise académica de 69. Já estava metido na vida académica e digamos que a canção vem por arrastamento, como um instrumento de combate. ${ }^{17}$

José Afonso:

Tudo se transformava em instrumento de luta e de intervenção. A tensão vivida, a energia nova exigiam uma poética nova, uma poética activa e útil [...] a vontade de mudar criava uma nova ética e precisava de uma nova estética [...] E nasceram as trovas. ${ }^{18}$

Manuel Alegre su Adriano Correia de Oliveira:

E a sua voz, a sua presença, as suas cantigas, são indissociáveis de toda a luta estudantil contra a ditadura e contra a guerra na década de 60 - e do que dela se prolongou até a vitória do 25 de Abril de 1974. A voz de uma geração de Abril...antes de Abril "Há sempre alguém que

${ }^{15}$ È celebre il caso della collaborazione tra Adriano Correia de Oliveira e il poeta Manuel Alegre, che darà vita a canzoni come Trova do vento que passa, vero e proprio inno della protesta studentesca. La canzone fu cantata anche dalla celebre fadista Amália Rodrigues.

${ }^{16}$ Da una conversazione con Eduardo Raposo, di cui leggiamo in Cantores de Abril, p. 26.

${ }^{17} \mathrm{Ib}$., p. 120.

${ }^{18} \mathrm{Ib}$., p. 241. 
resiste / há sempre alguém que diz não" - refrão da Trova do vento que passa, torna-se-ia um hino do movimento estudantil e um símbolo da resistência à ditadura. ${ }^{19}$

L'importanza strategica di questi cantanti era stata sancita dal Congresso da Oposição Democrática (Aveiro, 1973), a testimonianza del loro ruolo interno all'azione politica di movimenti e partiti (che fino al 25 aprile procederà di pari passo $)^{20}$. Tale importanza si manifesterà pienamente il 29 marzo 1974, nel partecipatissimo concerto al Coliseu dos Recreios di Lisbona, che vide intervenire quasi tutti i più importanti cantores de intervenção $o^{21}$.

Per dare la misura della partecipazione più che mai attiva dei cantanti impegnati portoghesi ai processi che portarono all'abbattimento del regime di Salazar e Marcelo Caetano, gioverà ricordare che sarà proprio la Grândola di Zeca Afonso (trasmessa alle ore 0.04. del 25 aprile 1975, a radio Renascença), a servire al Movimento dei Capitani come segnale per sancire il colpo di Stato che abbatteva il regime. Dice a questo proposito, José Niza:

Muita gente teve a noção exacta de que a viragem que se estava a dar era de esquerda porque as canções que passavam eram as nossas canções. A linguagem dos comunicados era extremamente prudente. 'As Forças Armadas' tomaram o poder [...] Não havia nenhum factor que desse um claro sinal político a não ser as canções. ${ }^{22}$

${ }^{19} \mathrm{Ib}$. , p. 26.

${ }^{20}$ Si distanzieranno nel periodo del PREC (Processo Revolucionário Em Curso), venuto a mancare il collante del nemico comune e dovendo entrare nel vivo della questione dell'assetto istituzionale da dare al Portogallo democratico.

${ }^{21}$ In quell'occasione Zeca canta, tra le altre, Grândola, già in precedenza registrata e distribuita in disco. Il testo fu pubblicato per la prima volta in Cantares, con prefazione di Manuel G. Simões, Tomar, Nova Realidade, 1966.

${ }^{22}$ E. Raposo, cit., p. 128. 
Le canzoni di protesta portoghesi non sono semplicemente la colonna sonora dei movimenti che portarono alla Rivoluzione dei garofani; esse ne costituiscono un momento di azione di importanza imprescindibile. ${ }^{23}$

Un ultimo dato merita di essere considerato. Mentre da noi la forte individualità connessa alla costruzione del personaggio del 'cantautore' impediva di fatto uno sganciamento delle personalità degli artisti dalle proprie produzioni ${ }^{24}$, in Portogallo le canzoni di lotta diventavano a tal punto terreno comune da non essere quasi più sentite come proprie dai loro creatori. Si veda a questo proposito l'illuminante testimonianza di Manuel Alegre:

Pela voz de Adriano Correia de Oliveira os poemas chegavam ao povo e ao país inteiro, a tal ponto que alguns desses poemas deixaram de ter autor para passarem a fazer parte da nossa memória comum e do nosso canto colectivo. Eu já não sinto como meus alguns dos poemas que o Adriano cantou. ${ }^{25}$

Ci si può chiedere, in conclusione, il perché di questa differenza abissale tra i due contesti. Dare una risposta esaustiva a questa domanda richiederebbe una riflessione che esula dalle possibilità di un articolo di breve respiro. Si può però azzardare un'ipotesi: può darsi che la percezione del Portogallo come 'eccezione' (in quanto 'dittatura') nel panorama occidentale abbia spianato la strada alla convergenza di differenti categorie sociali e professionali in una lotta che non dovrà porsi - come in Italia - l'annosa questione di trovare legittimazione all'interno di uno stato formalmente 'de-

${ }^{23}$ Numerosi i rapporti della censura in merito alla diffusione di canzoni che fiaccavano il morale delle truppe impegnate nella difesa dei territori ultramarini; quelle stesse truppe da cui sorgerà il Movimento dos Capitães.

${ }^{24}$ Migliori saranno, in questo campo, i risultati dei cantanti militanti, sempre comunque costretti entro il rischio di 'diventare cantautori'.

${ }^{25}$ E. Raposo, cit., p. 26. 
mocratico'? Sarebbe forse necessario, a questo punto, aprire una parentesi sul significato che il termine 'democrazia' ha assunto nel definire un tipo preciso di stato (quello socialdemocratico e liberista), proprio di molti Paesi occidentali, e su come movimenti come l'Autonomia si trovassero ad agire sul difficile terreno della decostruzione dell'identificazione di questo tipo di stato come 'democratico'. Ma questa è un'altra storia. 